\title{
ON FAMILIES OF FIBRED KNOTS WITH EQUAL SEIFERT FORMS
}

\author{
FILIP MISEV
}

\begin{abstract}
For every genus $g \geqslant 2$, we construct an infinite family of strongly quasipositive fibred knots having the same Seifert form as the torus knot $T(2,2 g+1)$. In particular, their signatures and four-genera are maximal and their homological monodromies (hence their Alexander module structures) agree. On the other hand, the geometric stretching factors are pairwise distinct and the knots are pairwise not ribbon concordant.
\end{abstract}

\section{INTRODUCTION AND SUMMARY OF RESULTS}

Baker proved in a recent note that two strongly quasipositive fibred knots $K_{0}, K_{1}$ must be equal if $K_{0} \#\left(-K_{1}\right)$ is a ribbon knot [4]. In view of Fox's famous slice-ribbon question, this leads to two equally intriguing alternative statements: either there exists a fibred knot of the form $K_{0} \#\left(-K_{1}\right)$ which is slice but not ribbon, or every knot concordance class contains at most one strongly quasipositive fibred knot.

We describe a simple and general construction proposed by Baader (compare [1]), which gives rise to infinite families of strongly quasipositive fibred knots having the same Seifert form. As a special case in which it is relatively easy to study the constructed knots in some detail, we obtain the following theorem.

Theorem 1. Let $g \geqslant 2$. There exists an infinite family of (pairwise nonisotopic) genus $g$ knots $K_{n} \subset S^{3}, n \in \mathbb{N}$, such that for all $n \in \mathbb{N}$

(1) $K_{n}$ is strongly quasipositive and fibred,

(2) $K_{n}$ has the same Seifert form as the torus knot $T(2,2 g+1)$.

To distinguish the constructed knots, we compare the geometric stretching factors of their monodromies, which can be calculated explicitly for $g=2$. The following corollary is an immediate consequence.

The author is supported by the Swiss National Science Foundation (\#168676). 
Corollary 1. Let $g \geqslant 2$. There exist infinitely many hyperbolic strongly quasipositive fibred knots of genus $g$, having

(1) maximal signature,

(2) periodic homological monodromy,

(3) isomorphic Alexander module structures.

Strongly quasipositive links were introduced and studied by Rudolph [16] and generalise links of singularities, positive braid closures, and positive links [17]. They are characterised as the boundaries of incompressible subsurfaces of positive torus link fibres [15], form a rather large class of knots representing every possible Seifert form [14, §3] and behave naturally with respect to fibredness: connected sum, plumbing and positive cabling preserve strong quasipositivity.

Families of fibred knots with equal Seifert forms have been studied before in connection with (ribbon) concordance. For example, Bonahon used Stallings twists to construct a family of fibred genus two knots $K_{n}$, $n \in \mathbb{N}$, whose homological monodromies are pairwise conjugate and such that $K_{n} \#\left(-K_{m}\right)$ is not a ribbon knot for $n \neq m$ (compare [6], see also [7]). However, these knots are not strongly quasipositive. In fact, a quasipositive surface cannot contain an essential zero-framed annulus [18], which is required for a Stallings twist. To prove that the knots $K_{n} \#\left(-K_{m}\right)$ are not ribbon, Bonahon applied the following criterion of Casson and Gordon [8, Theorem 5.1].

A fibred knot in a homology 3-sphere is homotopically ribbon if and only if its closed monodromy extends over a handlebody.

The notion of a knot being homotopically ribbon is a weakened version of ribbonness introduced by Casson and Gordon. If $S$ is a compact orientable surface with boundary, a diffeomorphism $\varphi: S \rightarrow S$ (fixing the boundary of $S$ pointwise) is said to extend over a handlebody if and only if there exists a three-dimensional handlebody $W$ such that $S \subset \partial W, \partial W \backslash S$ is a union of discs, and $\varphi$ is the restriction of a diffeomorphism of $W$.

Corollary 2. Let $K_{n}, n \in \mathbb{N}$, be the family of knots constructed in the proof of Theorem 1. The following holds for all $i, j \in \mathbb{N}, i \neq j$.

(1) $K_{i}$ and $K_{j}$ are not ribbon concordant,

(2) $K_{i}$ and $K_{j}$ are not homotopy ribbon concordant,

(3) the closed monodromy of $K_{i} \#\left(-K_{j}\right)$ does not extend over a handlebody,

(4) the smooth four-ball genus of $K_{i} \#\left(-K_{j}\right)$ is at most one. 


\section{FAMilies OF FIBRED KNOTS}

We first describe an explicit construction of a family of knots $K_{n}$, $n \in \mathbb{N}$, having the properties stated in Theorem 11. To prove that the knots are indeed pairwise nonisotopic, we compute the geometric dilatation $\lambda_{n} \in \mathbb{R}$ of the monodromy of $K_{n}$. For this purpose, we choose the construction in such a way that the monodromy is represented by a composition of two multi-twists, that is, products of Dehn twists on sets of disjoint curves.

Proof of Theorem 1 - Construction of the knots $K_{n}$. Let $S \subset S^{3}$ be the fibre surface of the $T(2,2 g)$ torus link, viewed as a plumbing of $2 g-1$ positive Hopf bands $H_{1}, \ldots, H_{2 g-1}$ according to the tree $\longrightarrow \bullet--\rightarrow \bullet$. We choose the plumbing order as follows. First let $S^{\prime}=H_{1} \# \ldots \# H_{g}$ be the connected sum of the first $g$ bands. The monodromy of $S^{\prime}$ is a composition of positive Dehn twists along $g$ pairwise disjoint simple closed curves, namely the core curves $\alpha_{1}, \ldots, \alpha_{g}$ of $H_{1}, \ldots, H_{g}$. Denote $\beta_{1}, \ldots, \beta_{g-1}$ the cores of the remaining Hopf bands $H_{g+1}, \ldots, H_{2 g-1}$, respectively. The latter may now be plumbed to $S^{\prime}$ from below such that $\alpha_{1}, \beta_{1}, \alpha_{2}, \beta_{2}, \ldots, \alpha_{g-1}, \beta_{g-1}, \alpha_{g}$ form a chain, that is, $\beta_{i}$ intersects each of its neighbours $\alpha_{i}$ and $\alpha_{i+1}$ transversely in one point (for all $i=1, \ldots, g-1$ ), and there are no other intersections (see Figure 1). Denote $S$ the resulting surface, which is indeed the fibre surface of
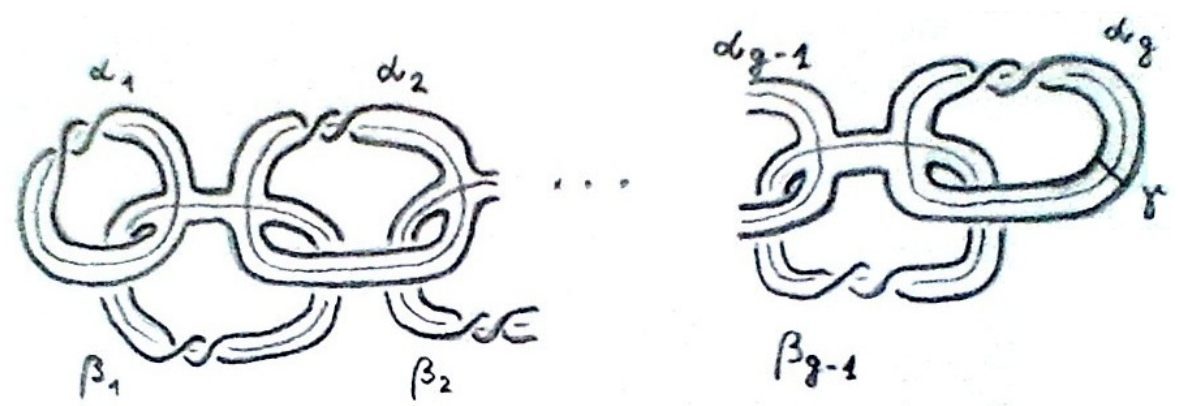

Figure 1. The fibre surface $S$ of the torus link $T(2,2 g)$ and core curves of the plumbed Hopf bands.

$T(2,2 g)$, since the plumbing order is irrelevant for arborescent Hopf plumbings. Now let $\gamma \subset H_{2 g-1} \subset S$ be a proper arc which intersects $\alpha_{g}$ transversely in one point and does not intersect any of the other curves. Further let $c \subset S$ be the boundary of a (small) regular neighbourhood of $\alpha_{g} \cup \beta_{g-1}$ in $S$ and denote $t_{c}$ the positive Dehn twist on $c$. Note that $t_{c}$ acts as the identity on homology since $c$ is nullhomologous in $S$, by construction (see Figure 2). For $n \in \mathbb{N}$, define the proper 


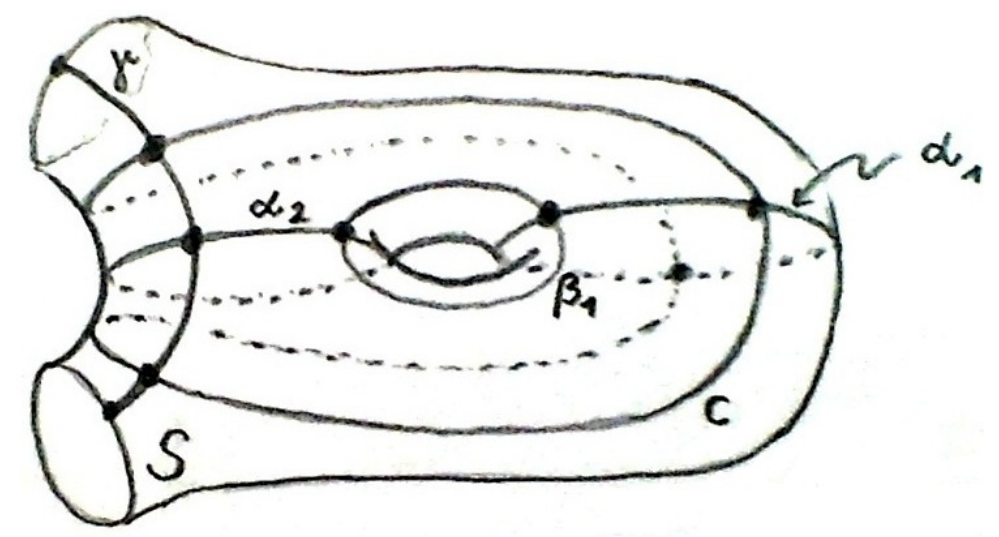

Figure 2. Position of the curves in the abstract surface $S$ for $g=2$.

arc $\gamma_{n}=t_{c}^{n}(\gamma)$. Finally, let $S_{n}$ be the surface obtained by plumbing a positive Hopf band $H_{2 g}$ along $\gamma_{n}$ to $S$ from below and denote $\beta_{g, n}$ the core curve of $H_{2 g}$ in $S_{n}$. The monodromy $\varphi_{n}: S_{n} \rightarrow S_{n}$ is given by

$$
\varphi_{n}=\left(t_{\beta_{g, n}} \circ t_{\beta_{g-1}} \circ \ldots \circ t_{\beta_{1}}\right) \circ\left(t_{\alpha_{g}} \circ \ldots \circ t_{\alpha_{1}}\right) \text {. }
$$

In other words, $\varphi_{n}$ is a composition of two positive multi-twists along systems of $g$ disjoint curves. Each complementary region of the union $\alpha_{1} \cup \ldots \cup \alpha_{g} \cup \beta_{1} \cup \ldots \cup \beta_{g-1} \cup \beta_{g, n}$ is either a boundary parallel annulus or a polygon which has at least three sides (corresponding to sub-arcs of the curves $\left.\alpha_{i}, \beta_{i}\right)$. Therefore, the curves fill up the surface $S$ and they realise their geometric intersection number (apply the bigon criterion of [9]). Moreover their union is connected. This fits the setting of Thurston's classical construction of invariant measured foliations for products of multi-twists (compare [23]). To compute the geometric dilatation $\lambda_{n}$ of $\varphi_{n}$, consider the $g \times g$ geometric intersection matrix

$$
N=\left[\begin{array}{ccccc}
1 & & & & \\
1 & 1 & & & \\
& \ddots & \ddots & & \\
& & 1 & 1 & 4 n \\
& & & 1 & 1
\end{array}\right]
$$

whose entry $N_{i j}$ is given by the number of intersection points (counted without sign) between $\alpha_{i}$ and $\beta_{j}$. Note that $\gamma \cap c$ and $\alpha_{g-1} \cap c$ consist of two points each, which implies that $\gamma_{n}$ (and therefore $\beta_{g, n}$ ) intersects $\alpha_{g-1}$ in $4 n$ points. Let $\mu_{n}$ be the largest eigenvalue of the symmetric 
matrix $N N^{\top}$, which is of the following form for $g \geqslant 3$.

$$
N N^{\top}=\left[\begin{array}{llllll}
1 & 1 & & & & \\
1 & 2 & & & & \\
& & \ddots & 1 & & \\
& & 1 & 2 & 1 & \\
& & & 1 & 2+16 n^{2} & 1+4 n \\
& & & & 1+4 n & 2
\end{array}\right]
$$

By a classical theorem of Geršgorin [10], the eigenvalues of a matrix $A$ are contained in the union of the discs in the complex plane with centers $A_{i i}$ and radii $\sum_{j \neq i}\left|A_{i j}\right|$ and if a disc is disjoint from the others, it must contain precisely one eigenvalue. For $A=N N^{\top}$, we see that the Geršgorin disc of radius $2+4 n$ centered at the largest diagonal entry $2+16 n^{2}$ is disjoint from all others if $n \neq 0$. Therefore

$$
16 n^{2}-4 n \leqslant \mu_{n} \leqslant 16 n^{2}+4 n+4, \quad \forall n \neq 0,
$$

hence the $\mu_{n}$ are pairwise distinct and unbounded. By Thurston's construction, the map $\varphi_{n}$ is pseudo-Anosov if and only if the following $2 \times 2$ matrix representing $\varphi_{n}$ is hyperbolic.

$$
T_{\underline{\beta}} \cdot T_{\underline{\alpha}}=\left[\begin{array}{cc}
1 & 0 \\
-\mu_{n}^{1 / 2} & 1
\end{array}\right] \cdot\left[\begin{array}{cc}
1 & \mu_{n}^{1 / 2} \\
0 & 1
\end{array}\right]=\left[\begin{array}{cc}
1 & \mu_{n}^{1 / 2} \\
-\mu_{n}^{1 / 2} & 1-\mu_{n}
\end{array}\right]
$$

Its eigenvalues are the geometric stretching factors $\lambda_{n}^{ \pm 1}$ of $\varphi_{n}$. A quick computation yields

$$
\lambda_{n}^{ \pm 1}=\frac{1}{2}\left(2-\mu_{n} \mp \sqrt{\mu_{n}^{2}-4 \mu_{n}}\right)
$$

(we chose the signs such that $\left|\lambda_{n}\right| \geqslant 1$ for $n \neq 0$ ). In particular, $\left|\lambda_{n}\right| \rightarrow \infty$ as $n \rightarrow \infty$ and the $\lambda_{n}$ are pairwise distinct. This implies that the knots $K_{n}:=\partial S_{n}$ are pairwise distinct and hyperbolic for $n \neq 0$. For $n=0$, we have $K_{0}=T(2,2 g+1), \varphi_{0}$ is a periodic mapping class and $\lambda_{0}$ is a root of unity.

By construction, $S_{n}$ is a plumbing of $2 g$ positive Hopf bands. Since a plumbing of positive Hopf bands is strongly quasipositive [16] and fibred [21], $K_{n}$ has the same properties, for all $n \in \mathbb{N}$.

It remains to show that the Seifert forms of the surfaces $S_{n}$ agree, up to the obvious identification of $H_{1}\left(S_{n}, \mathbb{Z}\right)=H_{1}(S, \mathbb{Z}) \oplus\left\langle\beta_{g, n}\right\rangle$ with $H_{1}\left(S_{m}, \mathbb{Z}\right)=H_{1}(S, \mathbb{Z}) \oplus\left\langle\beta_{g, m}\right\rangle$. The Seifert forms clearly agree on $H_{1}(S, \mathbb{Z})$, since the $S_{n}$ are all given by plumbing on $S$. Now let $\delta \subset S$ be a closed oriented curve and denote $\delta^{*}$ a slight push-off along the (positive or negative) direction normal to $S$. We have to prove that the linking numbers of $\beta_{g, n}$ and $\beta_{g, m}$ with $\delta^{*}$ agree. This follows from 
the fact that $\beta_{g, n}$ and $\beta_{g, m}$ bound discs whose interiors are disjoint from $S$, hence the linking numbers with $\delta^{*}$ are given by the algebraic intersection numbers between $\delta$ and $\beta_{g, n}, \beta_{g, m}$, respectively (compare Figure 3). The latter agree since the $\operatorname{arcs} \gamma_{n}$ and $\gamma_{m}$ are homologous in $S$ by construction.
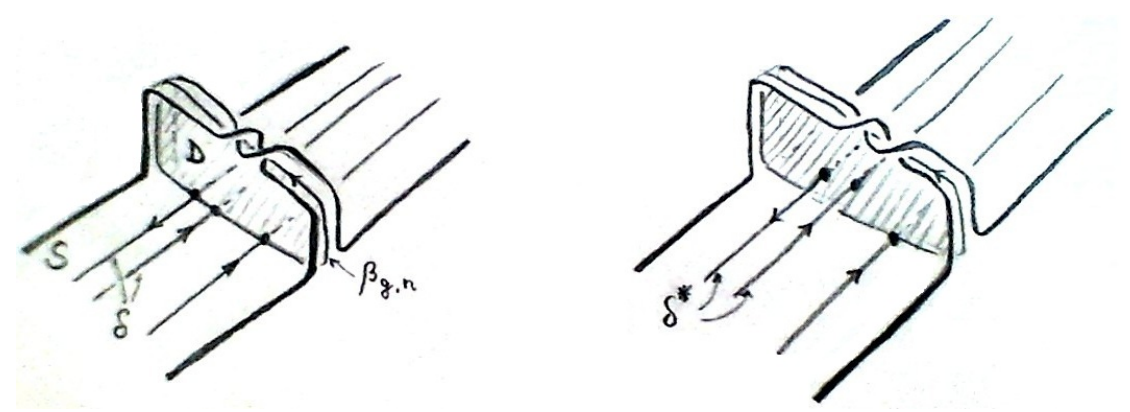

Figure 3 . The linking number of $\delta^{*}$ and $\beta_{g, n}$ equals the algebraic intersection number of $\delta$ and $\beta_{g, n} \cap S$.

Corollary 1. Let $g \geqslant 2$. There exist infinitely many hyperbolic strongly quasipositive fibred knots of genus $g$, having

(1) maximal signature,

(2) periodic homological monodromy,

(3) isomorphic Alexander module structures.

Proof. In the proof of Theorem 1 we constructed an infinite family of strongly quasipositive fibred genus $g$ knots $K_{n}, n \in \mathbb{N}$, such that $K_{n}$ is hyperbolic for $n \neq 0$ and has the same Seifert form as $K_{0}=T(2,2 g+1)$. Since $T(2,2 g+1)$ has maximal signature, this immediately implies (1). The homological monodromy $M$ of a fibre surface is related to its Seifert form $A$ by the formula $M=A^{-\top} A$ (compare [20, Lemma 8.3]). Hence $K_{n}$ has the same homological monodromy as $K_{0}$, which is periodic. Since the Alexander module structure of a fibred knot is determined by the homological action of the monodromy, this proves (2) and (3).

Corollary 2. Let $K_{n}, n \in \mathbb{N}$, be the family of knots constructed in the proof of Theorem 1 . The following holds for all $i, j \in \mathbb{N}, i \neq j$.

(1) $K_{i}$ and $K_{j}$ are not ribbon concordant,

(2) $K_{i}$ and $K_{j}$ are not homotopy ribbon concordant,

(3) the closed monodromy of $K_{i} \#\left(-K_{j}\right)$ does not extend over a handlebody,

(4) the smooth four-ball genus of $K_{i} \#\left(-K_{j}\right)$ is at most one. 
Remark. Note that homotopy ribbon concordance is (a priori) not a symmetric relation. Here, we say that two knots are homotopy ribbon concordant if one of them is homotopy ribbon concordant to the other.

Proof. By [11, Lemma 3.4], two homotopy ribbon concordant strongly quasipositive fibred knots have to be equal, hence (2). By a theorem of Casson and Gordon, (2) $\Leftrightarrow(3)$ for fibred knots (see [8, Theorem 5.1]). The implication $(2) \Rightarrow(1)$ holds for all knots. Assertion (4) essentially follows from the fact that the surface $S_{i} \#\left(-S_{j}\right)$ is given by plumbing two Hopf bands to the surface $S \#(-S)$, which is ribbon. More precisely, we can find a zero-framed unlink with unknotted components $L_{1}, \ldots, L_{2 g} \subset S_{i} \#\left(-S_{j}\right)$ which are realised as the embedded connected sum of the copies of the curves $\alpha_{k}, \beta_{k}$ in $S$ and $-S$, respectively. Cut $S_{i} \#\left(-S_{j}\right)$ along $L_{k}$ and glue two ribbon discs back in, for each $k$. Push the interior of the resulting surface slightly into the four-ball to obtain a ribbon surface of genus one.

\section{Further Properties of the KNOts $K_{n}$}

The subsequent statements refer to the family of knots $K_{n}$ of a fixed genus $g \geqslant 2$ constructed in the proof of Theorem 1 .

(1) Since the signature of a knot is a lower bound for the topological four-ball genus, we also have that $g_{4}^{t o p}\left(K_{n}\right)=g_{4}\left(K_{n}\right)=g\left(K_{n}\right)$. In particular, the $K_{n}$ are not slice (see also [18]).

(2) The equality of the Seifert forms also implies that the LevineTristram signature functions of the knots $K_{n}$ agree.

(3) The Alexander polynomial of $K_{n}$ is equal to the Alexander polynomial of $T(2,2 g+1)$ and the knots $K_{n} \#\left(-K_{m}\right)$ satisfy the Fox-Milnor condition.

(4) By work of Hedden [12], the concordance invariant $\tau$ is maximal for the knots $K_{n}$, that is, $\tau\left(K_{n}\right)=g\left(K_{n}\right)=g$. Moreover, the open book associated to $K_{n}$ supports the tight contact structure of $S^{3}$, so the geometric monodromy of $K_{n}$ is right-veering [13].

(5) The knots $K_{n}$ are all prime since their geometric monodromies are irreducible (they are pseudo-Anosov for $n \neq 0$ ).

(6) For $n \neq 0, K_{n}$ cannot be represented by a positive braid. In fact, positive braids of maximal signature have been classified by Baader [2]. They all have periodic (geometric) monodromy. All but finitely many of the $K_{n}$ (probably all but $K_{0}$ ) cannot be represented by a positive knot diagram. This follows from the fact that the number of positive knots of a fixed genus and fixed signature function is finite; compare [3, Proof of Theorem 1]. 
(7) Similarly, all but finitely many of the $K_{n}$ are non-alternating, since the number of alternating links of a given Alexander polynomial is finite by work of Stoimenow [22, Corollary 3.5].

(8) Except for a finite number of indices, the fibre surfaces of the $K_{n}$ cannot be Hopf-plumbed baskets (given by Hopf plumbing along arcs on a fixed disc [19]). In fact, the number of Hopf-plumbed baskets of a given genus is finite. This suggests that baskets should be thought of as rare exceptions among quasipositive fibre surfaces, at least from the point of view of general Hopf plumbing. See also [5].

\section{REFERENCES}

[1] S. Baader: Fibered knot with periodic homological monodromy, mathoverflow post, URL (version: 2013-09-30): http://mathoverflow.net/q/143579

[2] S. Baader: Positive braids of maximal signature, Enseign. Math. 59, no. 3-4 (2013), 351-358, arXiv:1211.4824.

[3] S. Baader, P. Dehornoy, L. Liechti: Signature and concordance of positive knots, (2015), arXiv:1503.01946v1.

[4] K. Baker: A note on the concordance of fibered knots, J. Topol. 9, no. 1, 1-4 (2016), arXiv:1409.7646v3.

[5] I. Banfield: Almost all strongly quasipositive braid closures are fibered, (2016), arXiv:1610.09664.

[6] F. Bonahon: Ribbon fibred knots, cobordism of surface diffeomorphisms, and pseudo-Anosov diffeomorphisms, Math. Proc. Camb. Phil. Soc. (1983), 94, $235-251$.

[7] F. Bonahon: Cobordism of automorphisms of surfaces, Ann. Sci. de l'E.N.S, serie 4, 16, no.2 (1983), 237-270.

[8] A. Casson, C. McA. Gordon: A loop theorem for duality spaces and fibred ribbon knots, Inventiones Math. 74 (1982), 119-137.

[9] B. Farb, D. Margalit: A Primer on Mapping Class Groups, Princeton University Press, (2012).

[10] S. Geršgorin: Über die Abgrenzung der Eigenwerte einer Matrix, Bulletin de l'Académie des Sciences de l'URSS, Classe des sciences mathématiques et na, no. 6 (1931), 749-754.

[11] C. McA. Gordon: Ribbon concordance of knots in the 3-sphere, Math. Ann. 257, no. 2 (Springer-Verlag, 1981), 157-170.

[12] M. Hedden: Notions of positivity and the Ozsváth-Szabó concordance invariant, J. Knot Theory Ramif. 19, no. 5, (2010), 617, arXiv:math/0509499.

[13] K. Honda, W. H. Kazez, G. Matić: Right-veering diffeomorphisms of compact surfaces with boundary, Invent. Math. 169 (2007), no. 2, 427-449, arXiv:math/0510639.

[14] L. Rudolph: Constructions of quasipositive knots and links, I, Nœuds, Tresses, et Singularités (ed. C. Weber), L’Enseign. Math. 31 (Kundig, Geneva, 1983), 233-246. 
[15] L. Rudolph: A characterization of quasipositive Seifert surfaces (Constructions of quasipositive knots and links, III), Topology, 31 (1992), no. 2, 231237.

[16] L. Rudolph: Quasipositive plumbing (Constructions of quasipositive knots and links, V), Proc. Amer. Math. Soc. 126, no. 1 (1998), 257-267.

[17] L. Rudolph: Positive links are strongly quasipositive, Geometry \& Topology Monographs, 2, Proceedings of the Kirbyfest (1999), 555-562.

[18] L. Rudolph: Quasipositivity as an obstruction to sliceness, Bull. Amer. Math. Soc., 29, no. 1 (1993), 51-59.

[19] L. Rudolph: Hopf plumbing, arborescent Seifert surfaces, baskets, espaliers, and homogeneous braids, Topology Appl. 116, no. 3 (2001), 255-277.

[20] N. Saveliev: Lectures on the Topology of 3-Manifolds: An Introduction to the Casson Invariant, De Gruyter, (2012).

[21] J. Stallings: Constructions of fibred knots and links, Algebraic and geometric topology, Proc. Sympos. Pure Math. 32 (1978), 55-60, Amer. Math. Soc., Providence, RI.

[22] A. Stoimenow: Knots of genus one, Proc. Amer. Math. Soc. 129, no. 7 (2001), 2141-2156.

[23] W.P. Thurston: On the geometry and dynamics of diffeomorphisms of surfaces, Bull. Amer. Math. Soc. 19, no.2 (1988).

Institut de Mathématiques I2M, Université AiX-Marseille, 39 Rue F. Joliot Curie, 13453 Marseille, France

E-mail address: filip.misev@univ-amu.fr 